2008-09-01

\title{
Correlation of Vibrational Intensity with Fluorescence Lifetimes in $\pi$ Conjugated Polymers
}

\author{
Luke O'Neill \\ Technological University Dublin, Luke.oneill@tudublin.ie \\ Patrick Lynch \\ Technological University \\ Mary McNamara \\ Technological University, Mary.McNamara@tudublin.ie
}

See next page for additional authors

Follow this and additional works at: https://arrow.tudublin.ie/materart

Part of the Physical Sciences and Mathematics Commons

\section{Recommended Citation \\ This Article is brought to you for free and open access by the Materials Synthesis and Applications at ARROW@TU Dublin. It has been accepted for inclusion in Articles by an authorized administrator of ARROW@TU Dublin. For more information, please contact arrow.admin@tudublin.ie, aisling.coyne@tudublin.ie, gerard.connolly@tudublin.ie.}

O'Neill, L. et al. (2008) Correlation of Vibrational Intensity with Fluorescence Lifetimes in $\pi$ Conjugated Polymers. Polymer, Vol. 49, Issue 19, pp. 4109-4114. 9th September. doi:10.1016/j.polymer.2008.07.035

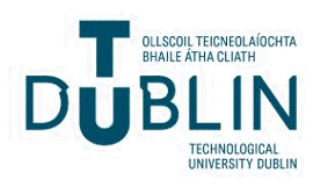




\section{Authors}

Luke O'Neill, Patrick Lynch, Mary McNamara, and Hugh Byrne

This article is available at ARROW@TU Dublin: https://arrow.tudublin.ie/materart/9 


\title{
Correlation of vibrational intensity with fluorescence lifetimes in $\pi$ conjugated polymers
}

\author{
Luke O’Neill $^{\text {a,c,*}, ~ P a t r i c k ~ L y n c h ~}{ }^{\text {b,c }}$, Mary McNamara ${ }^{\text {b,c }}$, Hugh J. Byrne ${ }^{\text {a,c }}$ \\ a School of Physics, Dublin Institute of Technology, Kevin Street, Dublin 8, Ireland \\ ${ }^{\mathrm{b}}$ School of Chemical and Pharmaceutical Sciences, Dublin Institute of Technology, Kevin Street, Dublin 8, Ireland \\ ${ }^{\mathrm{c}}$ Focas Research Institute, Dublin Institute of Technology, Camden Row, Dublin 8, Ireland
}

\section{A R T I C L E I N F O}

\section{Article history:}

Received 30 April 2008

Received in revised form 15 July 2008

Accepted 21 July 2008

Available online 26 July 2008

\section{Keywords:}

Polymers

Raman spectroscopy

Fluorescence lifetimes

\begin{abstract}
A B S T R A C T
A series of novel pi $(\pi)$ conjugated polymers, originating from the archetypical Polyphenylene vinylene, in which the phenyl units are successively replaced by the larger naphthyl and anthryl acene units, were previously found to have a well-defined relationship between their relative fluorescence yields and their vibrational characteristics, as determined by Raman spectroscopy. In this study the Strickler-Berg equation is used to probe the influence of continual substitution of higher order acene units into the conjugated backbone in terms of the variation of the radiative and non-radiative rates. The deconvolution of the radiative and non-radiative rates enables the correlation of the reduction of the Raman intensity and concomitant increase in the fluorescence yield with the reduction of the non-radiative rate. This confirms that the reduction of the non-radiative rate is the dominant process introduced by the vibrational confinement originating from systematic substitution of higher order acene units into the polymer backbone.
\end{abstract}

(c) 2008 Elsevier Ltd. All rights reserved.

\section{Introduction}

There has been an upsurge in the interest in conjugated polymers over the past two decades due to their unique optical and electronic properties [1-5]. Ease of fabrication, low cost, fast response time, wide viewing angle and light weight are just a few of the many properties that make conjugated polymers of great importance with a view to the advancement of opto-electronic devices [6-9]. Possibly the biggest advantage of conjugated polymer is the inherent ability to tune the optical and electronic properties by the subtle manipulation of the polymer backbone structure [10]. However, although much attention has been paid to control of the electronic absorption, little is known about structural control of the fluorescence efficiency or yield. This can be affected to potentially control either the radiative (electronic) or non-radiative (electron-vibrational) properties of the system.

It is well documented that the variation of the electronic properties of simple short chain molecules (oligomers) can be accurately described in terms of inverse conjugation length [1113]. This well-defined relationship has been extended to the vibrational characteristic of these short chain organic molecules [14]. Systematic studies of oligomeric series have thus contributed

\footnotetext{
* Corresponding author. School of Physics, Dublin Institute of Technology, Kevin Street, Dublin 8, Ireland. Tel.: +3531402 7906; fax: +35314027901.

E-mail address: luke.oneill@dit.ie (L. O’Neill).
}

greatly to the understanding of both the electronic and vibrational processes in more complex polymer systems. Although they may break down in the infinite-chain-length limit of polymeric systems, well-defined relationships shown to exist for simple conjugated molecules can be used as a guide to establish similar empirical relationships for more complex $\pi$ conjugated systems $[15,16]$.

In the series of novel PPV derivatives, shown in Fig. 1, it has been demonstrated that both the electronic and vibrational activities of these complex systems can be controlled and optimized by systematic variation of the polymer conjugated backbone $[17,18]$.

The vibrational intensity and fluorescence yield are seen to be well behaved with respect to systematic structural variation (Fig. 2), suggesting that limiting the vibrational activity of the polymer systems has the effect of reducing the non-radiative pathways. The goal of optimisation of the fluorescence yield, by minimisation the vibrational activity of the system, though a process of decoupling the vibrational frequencies has thus been shown to have merit. Regardless of the nature (electronic or vibronic) of the decoupling of the electronic and vibrational states, the end result is highly significant. Limitation of the vibrational activity of the systems becomes a viable route towards optimisation of the fluorescence yield. Thus structural modification can be used for optimisation of radiative processes and fluorescence yield, and structure-property relationships to guide synthetic strategies can be elucidated.

The aim of this communication is to report the progress in elucidating the decay processes of the polymers through investigation of their dynamics and associated lifetimes. The Strickler- 


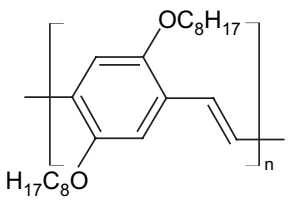

POPV (1)

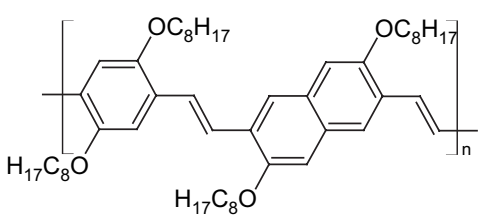

POPV-ONV (2)

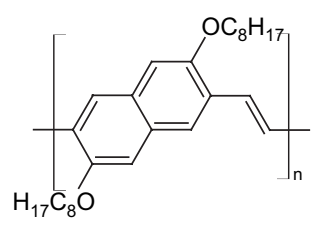

PONV (3)

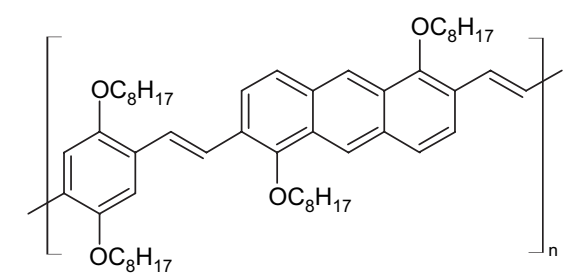

POPV-OAV (4)<smiles>CCCOc1c(/C=C/c2ccc3cc(CC)c(OCC)c(OCC)c3c2)ccc2cc(/C=C/c3ccc4c(OCC)c(C)ccc4c3OCC)ccc12</smiles>

PONV-OAV (5)

Fig. 1. Polymer structures 1-5.

Berg equation [19] will be used in conjugation with the electronic spectroscopy data to calculate the radiative and non-radiative rates for the progressive polymer systems.

$1 / \tau_{0}=2.880 \times 10^{-9} n^{2}\left\langle v_{\mathrm{f}}^{-3}\right\rangle A_{\mathrm{v}}^{-1} \frac{g_{\mathrm{l}}}{g_{\mathrm{u}}} \int \varepsilon \mathrm{d} \ln v$

where $n$ is the refractive index of the medium, $v_{\mathrm{f}}^{-3}$ is the integral over the fluorescence spectrum, $g_{1}$ and $g_{u}$ are the degeneracies of the lower and upper states, respectively, and $\varepsilon$ is the extinction coefficient.

The calculated non-radiative rates will be compared to the vibrational activity as measured by Raman spectroscopy in order to establish that this parameter can indeed be successfully used as a guide to the non-radiative decay rate in polymeric systems. The use of time correlated single photon counting (TCSPC) will be used to validate the calculated rates, and their structural dependencies. Thus the study will confirm that Raman spectroscopy can be used to probe structural variances in non-radiative rates, that these variances can be correlated with structural variances of fluorescence efficiencies, and that the strategy of reducing the electronvibrational coupling to optimise fluorescence efficiencies is valid.

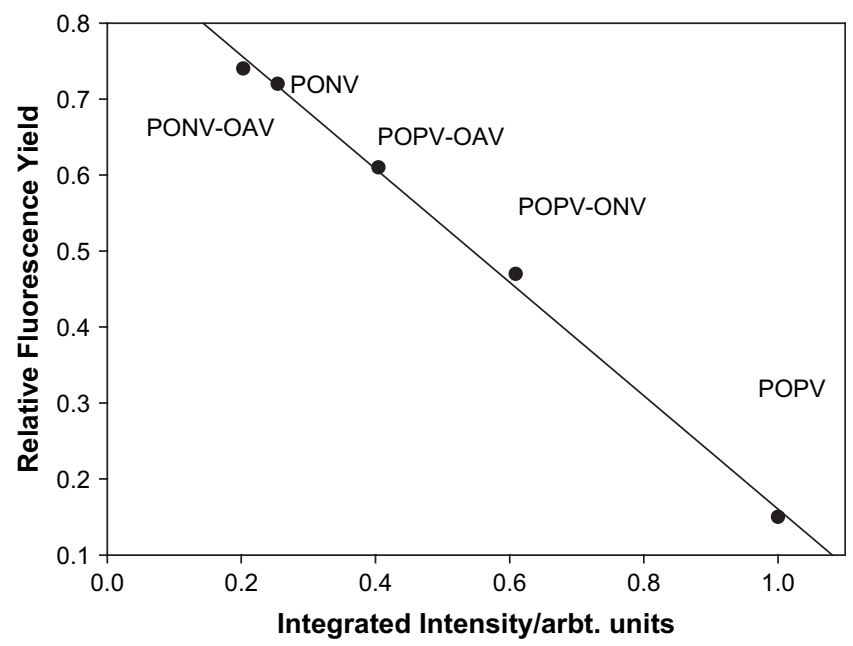

Fig. 2. Variation of the fluorescence yield with relative integrated Raman intensity; solid line is a linear fit.

\section{Experimental}

The polymer systems (Fig. 1$)$ investigated were Poly(2,5-bis- $(n-$ octyloxy)-phenylvinylene), POPV (1), Poly(2,5-bis(n-octyloxy)-1,4phenylene vinylene-1,5-bis(n-octyloxy)-2,6-naphthylene vinylene), POPV-ONV (2), Poly(2,6-bis-(n-octyloxy)-1,5-naphthylenevinylene), PONV (3), Poly(2,5-bis(n-octyloxy)-1,4-phenylene vinylene-co1,5-bis(n-octyloxy)-2,6-anthracene vinylene), POPV-OAV (4), and Poly(2,5-bis(n-octyloxy)-1,5-naphthylenevinylene-co-1,5-bis(n-octyloxy)-2,6-anthracene vinylene), PONV-OAV (5). Synthesis of the polymers 1-5, has been described elsewhere [20,21]. The polymers were prepared in chloroform solution of $\approx 10^{-6} \mathrm{M}$ for all fluorescence and absorption measurements.

Concentration dependent studies were undertaken to ensure that the samples were unaffected by aggregation [22]. Absorption spectroscopy was carried out using a Perkin Elmer Lambda 900 UV/ VIS/NIR absorption spectrometer. The luminescence measurements were performed using a Perkin Elmer LS55 luminescence spectrometer. These measurements were used to calculate relative fluorescence yields. All yields were calculated relative to MEH-PPV with a reported fluorescence yield value of 0.32 in solution [23].

Fluorescence lifetimes were measured using a computer controlled time correlated single photon counting spectrometer FL900 from Edinburgh Instruments. A nanosecond nF900 flashlamp excitation source using deuterium gas at a pressure of $\sim 0.40$ bar provided the fluorescence excitation pulses at $300 \mathrm{~nm}$. A Peltier cooled Hamamatsu R955 side-window photomultiplier tube (PMT) was used in an orthogonal geometry. All decay curves were corrected using a deconvolution with the instrument response function obtained using a scattering solution. The profile of the instrument response pulse had a FWHM of $\sim 1$ ns which was the detection limit of the system. All samples were run using a variation in concentrations from $10^{-4} \mathrm{M}$ to $10^{-9} \mathrm{M}$ at $300 \mathrm{~K}$ and in all cases the lifetime was found to be concentration independent within $0.1 \mathrm{~ns}$.

\section{Results}

In an effort to extend the empirical relationships shown to exist for the vibrational characteristics of the polymers' systems [18] it was decided to investigate the variation of the radiative and nonradiative rates, as the larger acene units were inserted in to the backbone, using the Strickler-Berg equation. It has been shown 

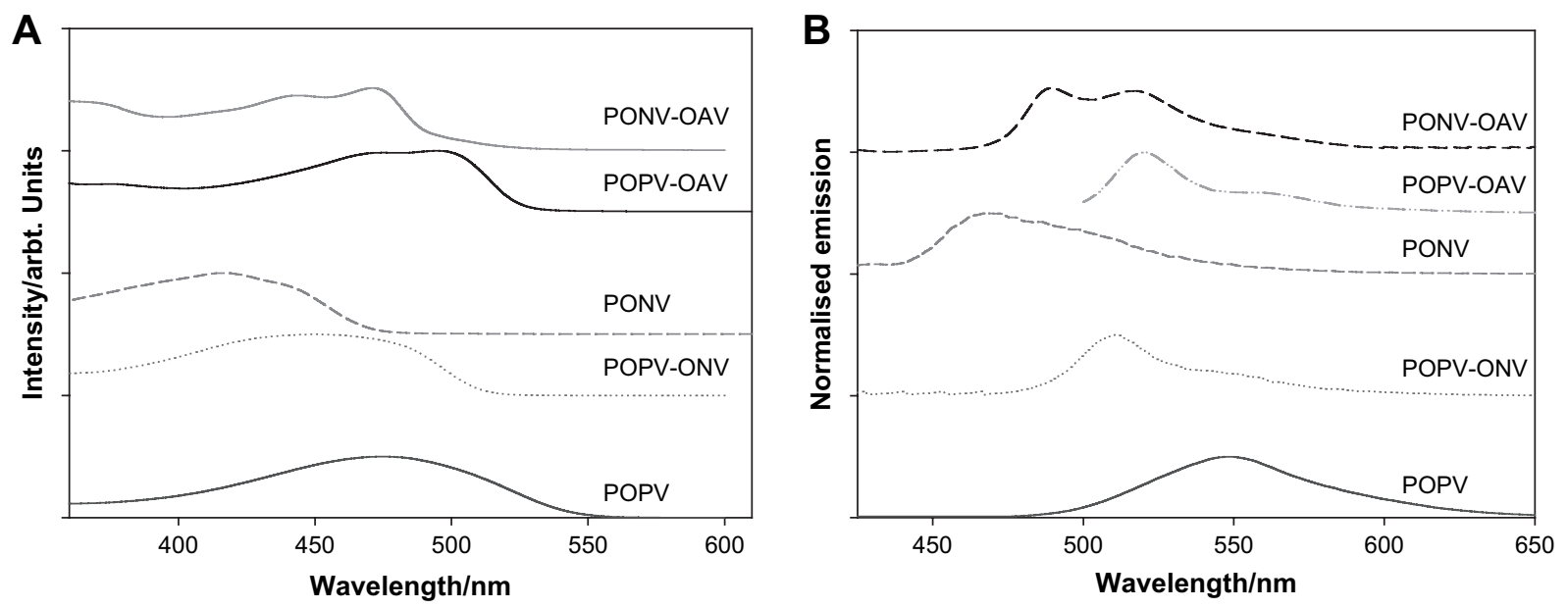

Fig. 3. Progression of polymer absorption spectra (A) and fluorescence spectra (B) from (1)-(5). Spectra are offset for clarity.

that the radiative rate $\left(K_{\text {rad }}\right)$ can be calculated using the absorption and emission spectra using the Strickler-Berg Eq. (1), [24,25].

It is assumed that the solvent has no optical dispersion. To calculate the radiative rate using the Strickler-Berg equation it is necessary to closely examine the UV and fluorescence data for the polymer series. The normalised absorption and fluorescence spectra of all polymers can be seen in Fig. 3. In POPV (1) the absorption spectrum shows peaks at $460 \mathrm{~nm}$ and $510 \mathrm{~nm}$, respectively. A significant hypsochromic shift is in evidence with continuing naphthyl substation. The POPV-ONV (2) and PONV (3) absorption peaks presented themselves at $460 \mathrm{~nm}, 480 \mathrm{~nm}$ and $440 \mathrm{~nm}, 420 \mathrm{~nm}$, respectively. With the extension of the polymer series from naphthyl substitution to an anthryl based series, POPVOAV (5) shows peak absorption features at $470 \mathrm{~nm}$ and $500 \mathrm{~nm}$, with the PONV-OAV (7), displaying prominent absorption features at $480 \mathrm{~nm}$ and $450 \mathrm{~nm}$. The same trends are mirrored in the fluorescence spectra as shown in Fig. 4. This variation of electronic parameters has been explained previously [17].

Using the UV and fluorescence data for the polymer series it was possible to use Eq. (1) to approximate the variation in the radiative rate $\left(K_{\mathrm{rad}}\right)$. Using the experimentally determined relative fluorescence yield and the calculated radiative rate it was possible to approximate the non-radiative rate using Eq. (2).

$\Phi_{\mathrm{f}}=\frac{K_{\mathrm{rad}}}{\left(K_{\mathrm{rad}}+K_{\text {non-rad }}\right)}$

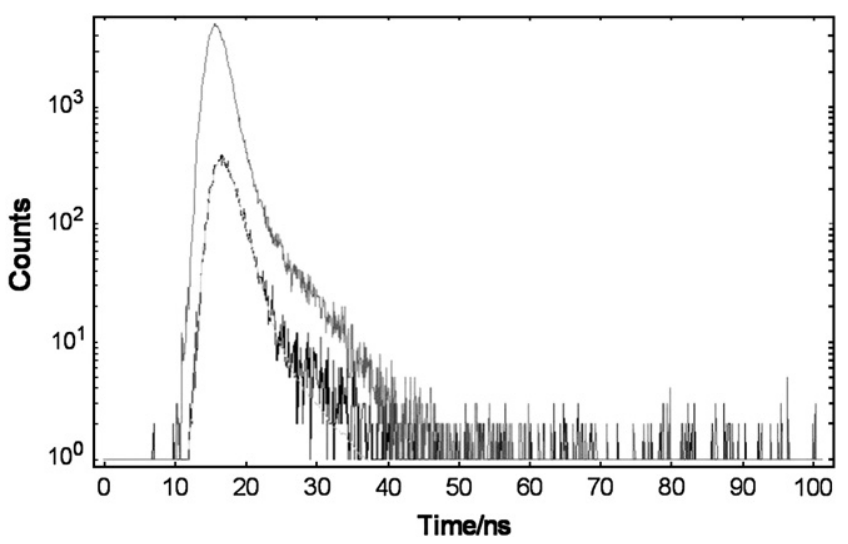

Fig. 4. Fluorescence decay profiles of POPV, IRF (grey), the POPV decay (black) and the light grey line indicating a deconvoluted fit.
The calculated values for the radiative and non-radiative rates can be found in Table 1 . The relative fluorescence yield values are also presented.

From Table 1 it is apparent that as the larger more conjugated units are substituted into the polymer chain there is a fluctuation in the radiative rate coupled with a significant decrease in the nonradiative rate. The design ethos of this study was to decrease the non-radiative decay and hence optimise the fluorescence yield though structural manipulation of the avenues if vibrational relaxation. Although both the radiative and non-radiative rates are influenced by the systematic substitution of higher acene units into the $\pi$ system, the radiative rate remains relatively constant, whereas the non-radiative rate shows a consistent reduction, by more than an order of magnitude.

This is a clear indication that the dominant change, introduced by the systematic alteration of the $\pi$ conjugated backbone, is the reduction of the non-radiative rate. This result correlates well with the reduction of the relative Raman intensity and the increase in the fluorescence yield published previously [18].

In order to further explore the tentative relationship suggested through the calculation of the radiative rates using the StricklerBerg equation it was decided to experimentally determine the fluorescence lifetime and compare this to the calculated lifetime. To this end it was decided to employ time correlated single photon counting (TCSPC) to determine the fluorescence lifetimes. The resultant decay curves will establish the variation of the fluorescence lifetime as the backbone is systematically varied. The dominant non-radiative rate being systematically reduced should permit a longer lived excited state.

In an effort to accurately gauge the changes in the decay dynamics due to the systematic changes in the backbone of the PPV derivatives, it was first necessary to examine the decay profile of the basic building block, the polymer POPV (1). The decay profile of POPV is shown in Fig. 4. The emission of POPV was recorded at $550 \mathrm{~nm}$, which had been established as the peak emission

Table 1

Polymer photophysical rates: $\Phi_{\mathrm{f}}^{*}$ relative fluorescence yield, $K_{\mathrm{rad}}$ radiative rate and $K_{\text {non-rad }}$ non-radiative rate

\begin{tabular}{llll}
\hline Polymer name & $\Phi_{\mathrm{f}}^{*}$ & $K_{\text {rad }} / \mathrm{S}^{-1} \times 10^{7}$ & $K_{\text {non-rad }} / \mathrm{S}^{-1} \times 10^{7}$ \\
\hline POPV & 0.15 & 5.0 & 28.3 \\
POPV-ONV & 0.47 & 5.4 & 6.1 \\
POPV-OAV & 0.61 & 7.0 & 4.5 \\
PONV & 0.72 & 7.4 & 2.9 \\
PONV-OAV & 0.74 & 5.6 & 1.9 \\
\hline
\end{tabular}



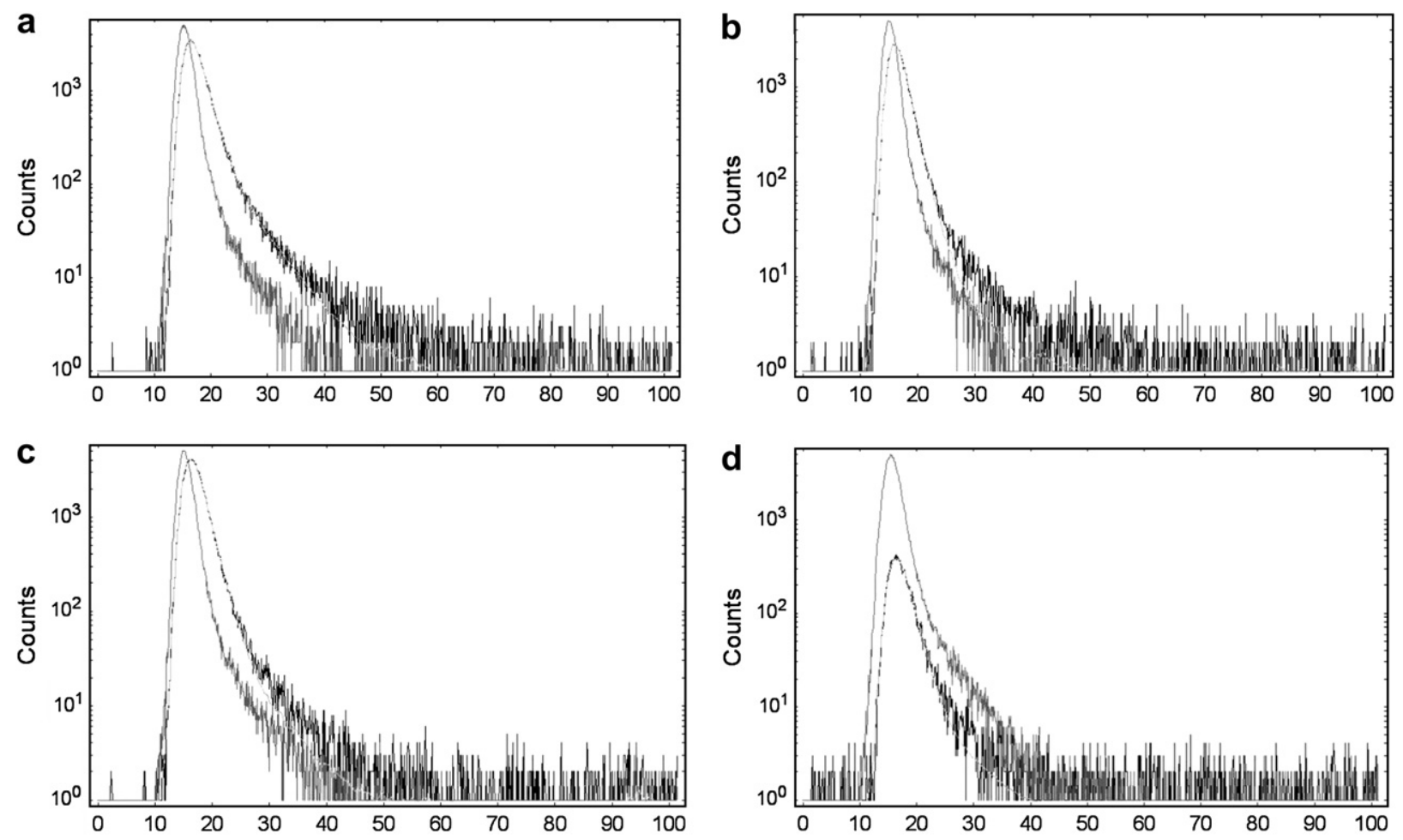

Fig. 5. Fluorescence decay profiles of PONV-OAV (a), POPV-OAV (b), PONV (c), and POPV-ONV (d); instrument response function (grey), decay profile (black).

wavelength [17]. A deconvolution fit gives a lifetime of $1.16 \mathrm{~ns}$ for the POPV polymer. In Fig. 5 the decay curves for PONV-OAV (a), POPV-OAV (b), PONV (c), and POPV-ONV (d) are shown. In all cases a single exponential function was fitted to the decay curves to obtain a value for the fluorescence lifetimes.

The fluorescence lifetimes of the aforementioned polymers were calculated to be $1.27 \mathrm{~ns}, 1.34 \mathrm{~ns}, 1.47 \mathrm{~ns}$ and $1.50 \mathrm{~ns}$ for POPVONV (d), POPV-OAV (b), PONV (c), and PONV-OAV (a), respectively. A significant trend emerges from the fitting to the decay data, as the larger and more electron withdrawing constituents are introduced into the polymer chain the lifetime progressively lengthens.

All fluorescence lifetimes measured can be found in Table 2 . The prototype polymer, POPV, has a fluorescence lifetime of 1.16 ns. The introduction of a naphthyl unit into the backbone shifts the fluorescence lifetime to a higher value of $1.27 \mathrm{~ns}$ and further systematic substitution leads to lifetimes of $1.34 \mathrm{~ns}$ and $1.47 \mathrm{~ns}$ for POPV-OAV and PONV, respectively. This trend is continued with the longest measured lifetime found to be $1.50 \mathrm{~ns}$, for PONV-OAV. The extension of the fluorescence lifetime further strengthens the assertion that the non-radiative rate is the dominating factor in the deactivation of the exciting state as shown through the Strickler-Berg calculations. This is further evidenced that the reduction in vibrational freedom works to limit the avenues for non-radiative decay though phonon confinement.

Using the Strickler-Berg equation the mean lifetime was calculated using absorption and emission spectra for the polymers.

\section{Table 2}

Fluorescence life $\left(\tau_{\mathrm{fl}}\right)$, calculated fluorescence life $\left({ }^{*} \tau_{\mathrm{fl}}\right)$ and relative fluorescence yield $\left(\Phi_{\mathrm{f}}^{*}\right)$

\begin{tabular}{llcc}
\hline Polymer name & Experimental $\tau_{\mathrm{fl}} / \mathrm{ns}$ & Calculated $^{*} \tau_{\mathrm{fl}} / \mathrm{ns}$ & $\Phi_{\mathrm{f}}^{*}$ \\
\hline POPV & 1.16 & 3.0 & 0.15 \\
POPV-ONV & 1.27 & 8.7 & 0.47 \\
POPV-OAV & 1.34 & 8.8 & 0.61 \\
PONV & 1.47 & 9.6 & 0.72 \\
PONV-OAV & 1.50 & 13.3 & 0.74 \\
\hline
\end{tabular}

From these spectra the mean value of $v_{\mathrm{f}}^{-3}$ can be ascertained and the lifetime approximated. It is evident that the Strickler-Berg equation significantly overestimates the fluorescence lifetime of the polymers under scrutiny; however, the trend established with the calculated data is mirrored in the experimental values. This overestimation can be attributable to refractive index variation and also allowing for the fact that the Strickler-Berg equation assumes the geometry of the molecule stays the same as it goes from the ground to the excited state. As can be seen from Fig. 2 the mirror symmetry is broken when comparing the fluorescence and the absorption spectra suggesting a change in structural geometry upon excitation.

The calculated non-radiative rate is plotted as a function of Raman intensity in Fig. 7. It is clear that as the Raman intensity is decreased there is a marked and continual reduction of the nonradiative rate. This progressive reduction can be best fit by a single exponential. This confirms the hypothesis that as the vibrational intensity is reduced the electron phonon coupling is reduced and hence the avenues for non-radiative decay are restricted. Thus it is evident that the reduction in the Stokes shift would correspondingly be well correlated with the non-radiative rate. This is as expected as it has been shown that the Stokes shift is well correlated with integrated Raman intensity, and thus can be used as a crude measurement of the vibrational activity of the system [18].

As can be seen from Table 1 and from Figs. 6 and 7 the radiative and non-radiative rates calculated from the Strickler-Berg equation agree with the previous assumption as to the reduction of the vibrational intensities having a significant effect on the non-radiative decay dynamics. Furthermore the fluorescence lifetimes calculated from the rates are consistent with the documented PPV derivative lifetime measurements [26]. The polymer fluorescence lifetimes can be calculated to be in the order of nanoseconds as is expected of substituted PPV systems [27].

The proposed direct correlation between the vibrational activity and the non-radiative rate present in the polymer systems means that the vibrational intensity can be used as an accurate gauge of 


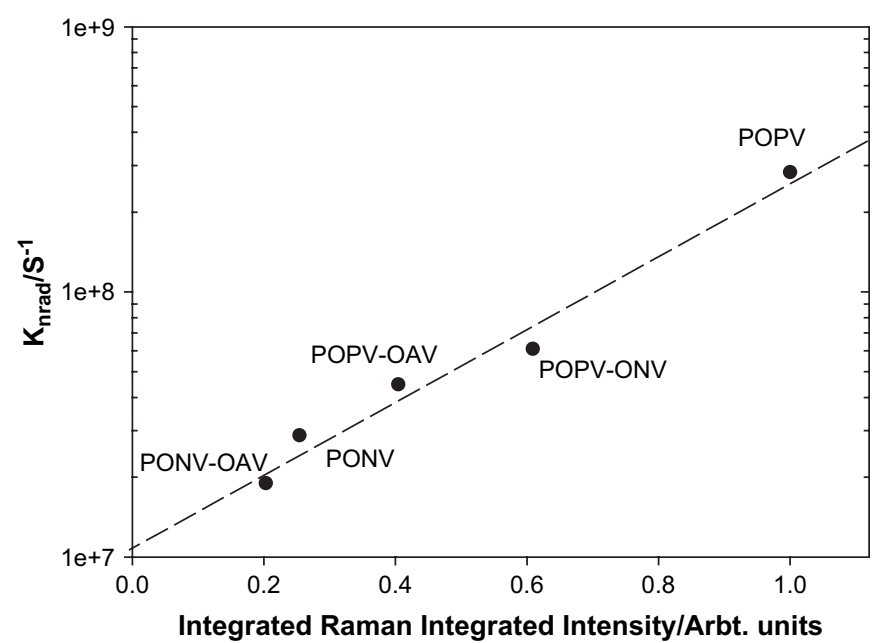

Fig. 6. Plot of calculated non-radiative rate against Raman intensity; line is guide for the eye.

the non-radiative processes. A graph of the inverse fluorescence lifetime against Raman intensity should yield a linear relationship if this is indeed the case. It can be seen from Fig. 8 that the graph does indeed show a well-defined almost linear trend. If the assumption that the non-radiative rate is the dominant factor in the de-population of the excited states then the reduction of the non-radiative rate through phonon confinement would lead to the longer lived excited state and thus a larger fluorescence lifetime as is borne out in Table 2.

This is a simple explanation, which assumes that the triplet yield is negligibly small and that in the variation of the backbone structure the most significant effect is in the reduction of the nonradiative rate. This assumption is not unfounded as it has been shown that contrary to the trend shown in acene oligomers [14], a plot of the vibrational intensity against the fluorescence yield gives a well-defined relationship that is almost linear [18]. This would suggest that it is the non-radiative yield that is dominating the fluorescence yield. It has been shown that the triplet yield in PPV is as low as 0.06 [28], which is negligible when compared to the $\sim 0.80$ triplet yield exhibited by the shorter oligomers. Hence the triplet yield can be considered as a minor factor in comparison to the non-radiative decay through the vibrational channels in the extended backbone of the polymer. To further reinforce this point it has been reported in MEH-PPV that of the total non-radiative yield of 0.76 , the triplet contribution is only responsible for 0.01 [29]. It

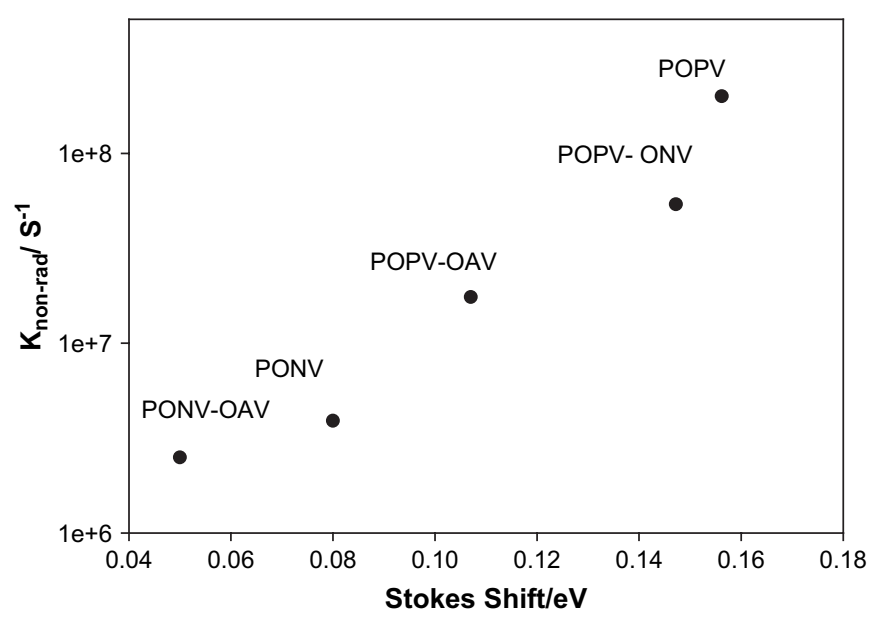

Fig. 7. Plot of calculated non-radiative rate against Stokes shift; line is guide for the eye.

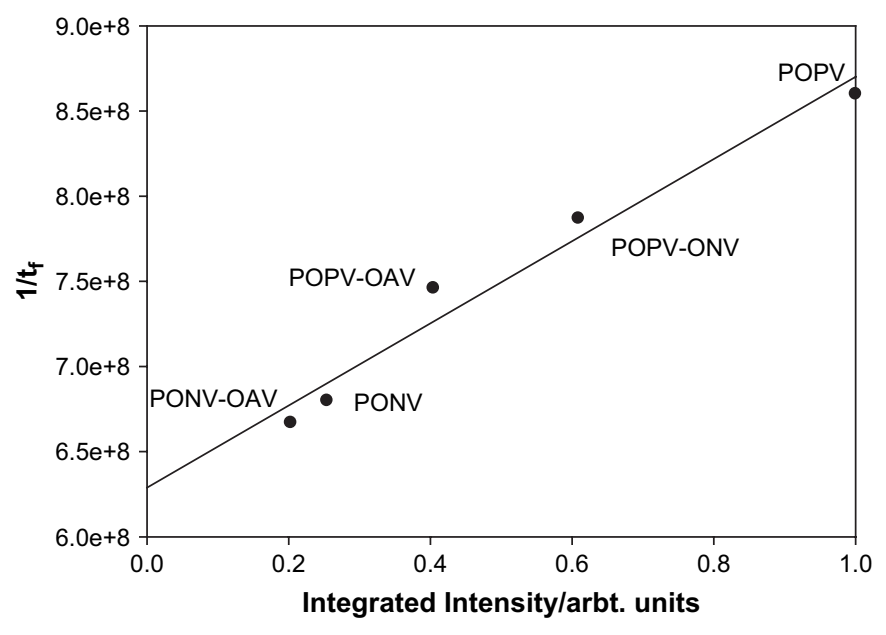

Fig. 8. Plot of integrated inverse fluorescence lifetime against Raman intensity.

has also been documented that for PPV derivatives, such as MEHPPV and cyano substituted PPVs, the inclusion of electron withdrawing side chains serves to prolong the fluorescence lifetime [30] as is the characteristic shown in our acene substituted series. The triplet yield for the acene series itself is well documented [31] and it has been seen that intersystem crossing dominates the structural variation of the fluorescent yield in the shorter chain molecules. The inclusion of the longer chain, lower triplet yield acene units into the backbone should result in a further reduction of the triplet contribution. It is therefore considered justifiable to neglect the intersystem crossing contributions to the fluorescent lifetime. Hence the domination of the non-radiative decay in the determination of the fluorescence life should result in a longer lifetime if the avenues of non-radiative decay are reduced. This account of the decay dynamic is in full agreement with the calculated non-radiative and radiative rates and the fluorescence lifetimes measured.

The establishment of well-defined vibrational characteristics exist for $\pi$ conjugated polymers [18] has led to a degree of control of the fluorescent yield. Both the calculated non-radiative rates and the fluorescence lifetimes have confirmed that the tailoring of the backbone can be used as an efficient tool for the reduction of the non-radiative avenues of decay.

\section{Conclusions}

The non-radiative and radiative rates calculated using the Strickler-Berg equation have shown that the systematic tailoring of the $\pi$ conjugated backbone is an realistic tool to minimise the nonradiative decay rate. The calculated radiative rate has also been shown to have a well-defined trend with increasing acene substitution; the subsequent calculation of the non-radiative rate also allows the direct observation of the variation of the non-radiative rate and its correlation with Raman intensity. It is shown that the non-radiative rate varies in a well-defined manner and it is apparent that the large (greater than factor of 10) change in the non-radiative rate is the dominant process compared to the random fluctuation of the radiative rate. This confirms that the Raman intensity can be used as an accurate measure of the nonradiative rate. A reduction in the non-radiative rate was similarly mirrored with a concomitant reduction in the Raman vibrational intensity. There is also a direct correlation between the extension of the fluorescence lifetime and the reduction of the vibrational activity of the systems. This subtle manipulation of the polymer backbone through systematic insertion of larger acene units has been shown to significantly reduce the non-radiative rate and hence ultimately affects a larger increase in the fluorescence yield. 
The results of the TCSPC reinforce the relationships proposed in the vibrational study [18] and also confirm the results of the Strickler-Berg equation, thus further illustrating the use of the tailorability of the vibrational characteristics as a tool to optimise the fluorescence yield.

\section{Acknowledgements}

The FOCAS Institute is funded under the National Development Plan 2000-2006 with assistance from the European Regional Development Fund. L. O'Neill acknowledges DIT Scholarship support.

\section{References}

[1] Schwartz BJ, Hide F, DiazGarcia MA, Andersson MR, Heeger AJ. Philos Trans R Soc London Ser A 1997;355:775.

[2] Sauteret C, Hermann JP, Frey R, Pradere F, Ducuing J, Baughman RH, et al. Phys Rev Lett 1976;36:956.

[3] Rustagi KC, Ducuing J. Opt Commun 1974;10(3):258.

[4] Burroughes JH, Bradley DDC, Brown AR, Marks RN, Mackay KD, Friend RH, et al. Nature 1990;347:539.

[5] Friend RH, Gymer RW, Holmes AB, Burroughes JH, Marks RN, Taliani C, et al. Nature 1999;397:121.

[6] Sirringhaus H, Tessler N, Friend RH. Science 1998;280:1741.

[7] Andersson MR, Thomas O, Mammo W, Svensson M, Theander M, Inganas O. J Mater Chem 1999;9:1933.

[8] Tsuboi T, Tanigawa M, Kawami S, Tsuji T. Curr Appl Phys 2005;5:47.
[9] Gu G, Parthasarathy G, Tian P, Burrows PE, Forrest SR. J Appl Phys 1999; 86:4076.

[10] Wenz G, Muller MA, Schmidt M, Wagner G. Macromolecules 1984;17:837.

[11] Meier H. Angew Chem Int Ed 2005;44:2482.

[12] Wolhlgonannt M, Jiang XM, Vardony ZV, Janssen RAJ. Phys Rev Lett 2002;88: 197401.

[13] Byrne HJ, Blau W, Giesa R, Schulz RC. Chem Phys Lett 1990;167:484.

[14] O'Neill L, Byrne HJ. J Phys Chem B 2005;109:12685.

[15] Angiolini L, Benelli T, Giorgini L, Salatelli E. Polymer 2006;47:1875.

[16] Martin RE, Diederich F. Angew Chem Int Ed 1998;38:1350.

[17] O'Neill L, Lynch P, McNamara M, Byrne HJ. J Phys Chem A 2007;111:299.

[18] O’Neill L, Lynch P, McNamara M, Byrne HJ. J Phys Chem B 2007;111:7999.

[19] Strickler SJ, Berg RA. J Chem Phys 1962;37:814-22.

[20] Lynch P, O’Neill L, McNamara M, Byrne HJ. Marcomolecules 2007;40:7895.

[21] Maier S. Doctoral Thesis, University of Dublin Trinity College; 2000.

[22] O'Neill L. Doctoral Thesis, Dublin Institute of Technology; 2006.

[23] Zheny M, Ba F, Zhu D. Polym Adv Technol 1999;10:476.

[24] Hsu JH, Hayash M, Lin S-H, Fann W, Rothberg LS, Peng G-Y, et al. J Phys Chem B 2002;106:8582.

[25] Grummt UW, Pautzsch T, Birckner E, Sauerbrey H, Utterodt A, Neugebauer U, et al. J Phys Org Chem 2004;17:199.

[26] Kyllo EM, Gustafson TL, Wang DK, Sun RG, Epstein AJ. Synth Met 2001; 116:189.

[27] Heller CM, Campbell IH, Laurich BK, Smith DL, Bradley DDC, Burn PL, et al. Phys Rev 1996;54:5516.

[28] Candeias LP, Wildeman J, Hadziioannou G, Warman JM. J Phys Chem B 2000; 104:8366.

[29] Burrows HD, Seixas de Melo J, Serpa C, Arnaut LG, Monkman AP, Hamblett I, et al. J Chem Phys 2001;115:20.

[30] Samuel DW, Rumbles G, Collison CJ. Phys Rev B 1995;52:11573.

[31] Birks B. Photophysics of aromatic molecules. Wiley Interscience; 1970. 